\title{
INVESTIGACIÓNY DESARROLLO (I+D) Y LA DEPENDENCIA ECONÓMICA
}

\author{
CARLOS CASTRO QUESADA \\ Universidad Tecnológica Costarricense \\ cjcquniversidad@gmail.com
}

\section{RESUMEN}

La incursión de la red Internet en el campo científico se está convirtiendo en una seria amenaza para el -hasta hoy-dominio casi exclusivo de los países desarrollados y (como principal agente económico) de las empresas multinacionales. El establecimiento, por parte de los países en vías de desarrollo, de una incipiente pero importante red científica y tecnológica; hace que, por primera vez en la historia, la comunicación especializada, entre profesionales de casi todas las ramas de la actividad científica, sea cada vez más frecuente. Esta es una nueva oportunidad que se le abre al mundo subdesarrollado para aspirar a ser más competitivo en el área de la Investigación y el Desarrollo (I+D). No obstante, queda claro que la parte más difícil (por sus elevados costos) es la del desarrollo de nuevos productos y servicios; siendo precisamente este un campo en el que los países desarrollados (por sus altas posibilidades de inversión) seguirán teniendo una posición de privilegio. El presente ensayo, plantea la posibilidad de transformar (para el mundo subdesarrollado) el concepto de I+D por el de Investigación e Innovación (I+I), sacándole así mayor provecho a lo exiguo de los recursos financieros que se pueden destinar a la investigación, desarrollo e innovación científica.

PALABRAS CLAVE: CIENCIA, INVESTIGACIÓN, DESARROLLO, INNOVACIÓN Y CRECIMIENTO ECONÓMICO.

\begin{abstract}
The incursion of the WWW system, or the Internet System, in scientific fields, has become an increasing threat for an almost monopoly of the developed countries, and among them, the multinationals enterprises An incipient but nevertheless important scientific and technological net works was established by the under-developed countries, for the first time in history; specialized communication has been more frequent between specialists of almost all branches of scientific activities. This is a new opportunity that has opened to the under-developed world, to aspire to be more competitive in the areas of I+R. Nevertheless, it is clear that the most difficult part, because of its cost, is the development itself, field in which the developed countries, due to their high possibilities, will continue to have mayor advantages. That is why, in this essay is suggested the possibility, to transform the I+R for that: I+I (investigation and innovation), for the under-developed world. To this matter it's imperative, for the under-developed countries, to withdraw from the limited financial resources; that can be, destined to investigation, innovation and scientific investigation.
\end{abstract}

KEYWORDS: SCIENCE, INVESTIGATION, DEVELOMENT, INNOVATION AND ECONOMIC GROWTH.

\section{INTRODUCCIÓN}

En una sociedad abierta y globalizada, no se puede pretender mantener a la ciencia (o a una parte de ella) aislada dentro de una "torre de marfil"; es un empeño inútil que no hace más que prolongar 
la agonía hacia la irrelevancia, precisamente por su aislamiento.

Los hasta ahora beneficiados por tal aislamiento, han sido sin duda los países desarrollados y, como parte fundamental de su organización económica , las empresas multinacionales o "globalizadas" en razón de sus casi infinitas posibilidades económicas.

No obstante, el aislamiento científico empresarial, tiene su principal amenaza en la extensión casi universal de la Red Internet y, dentro de ella, en el desarrollo de redes tecnológicas y científicas en las que la investigación y la innovación de carácter científico e incluso tecnológico; corre libremente en un verdadero "mundo sin fronteras".

Aunque la diferencia de lo que se invierte en I+D, entre los países desarrollados y los países en vías de desarrollo, sigue siendo abismal; algunos de estos últimos países han optado por un incremento significativo en investigación científica con resultados verdaderamente sorprendentes.

El presente trabajo pretende auscultar las posibilidades reales que la I+D le ofrece a los países en vías de desarrollo, de encontrar una senda segura y definitiva para emprender con paso firme, el difícil y hasta ahora negado, camino hacia el desarrollo.

\section{Nuevo paradigma}

Tapscott y Williams (2007), autores del revolucionario libro "Wikinomics", definen un nuevo paradigma científico basado en la colaboración utilizando, para tal efecto la ventaja que supone la extensión casi universal de la red Internet.

El surgimiento de esta red ha provocado profundos cambios en la economía global siendo, entre otros muchos, el desarrollo de nuevos modelos de producción basados en la cooperación. Trabajadores de todas partes del mundo logran elevar su productividad gracias al intercambio de conocimientos con otros individuos de su propia área de actividad laboral quienes se en- cuentran ubicados "extra-muros" con respecto a su lugar de trabajo.

Es así como las "cadenas de suministro" se nutren con los aportes de socios que pueden estar localizados en cualquier rincón del planeta pero que logran comunicarse a través de la red.

El desarrollo de esta nueva ciencia de la cooperación universal es lo que Taspscott y Williams (2007) han denominado "wikinomía" (wikinomics en el original inglés) que se fundamenta en "nuevos principios de competitividad como la apertura, la comunicación entre iguales, el compartir y la actuación global" (p. 18).

Parte importante de esta nueva conceptualización del desarrollo empresarial es la creación de "valor agregado" a través de la investigación y la innovación científicas. Son millones de personas alrededor del todo el mundo que, con sus aportes, están promoviendo el surgimiento de nuevos bienes y servicios que vienen a competir con sus similares producidos por las grandes empresas multinacionales.

Como resultado, la capacidad de innovación estará distribuida por todo el mundo sin hacer diferenciación alguna entre países ricos y pobres pues solo bastará con tener una computadora y una conexión a Internet para poder aportar algo que se pueda convertir en "valor agregado" a través del desarrollo de mejoras en los bienes y servicios (o sus procesos) ya existentes, o en la creación de bienes y servicios completamente nuevos.

Ante el surgimiento de la wikinomía, las grandes empresas, utilizando el principio de economías de escala, se prestan a ir abandonando su investigación en laboratorios de su propiedad y ubicados en sus propias instalaciones, para centrarse en este nuevo tipo de investigación que puede tener lugar en cualquier rincón del planeta, de modo que sea factible -a mediano plazo- aprovechar las redes de colaboración 
con individuos, centros de investigación y otras empresas especializadas, que pueden, como ya se dijo, estar ubicadas en cualquier parte de la tierra.

Obviamente que estas redes no se basan ya en los acuerdos tradicionales fundamentados en la protección estricta de la propiedad intelectual y los derechos de autor y más bien se centran en acelerar los procesos de descubrimiento científico y su transformación, por ellos mismos o por terceros, en nuevos productos y servicios, mediante la utilización de agentes independientes.

Se estaría pasando así, de una estrategia defensiva basada en departamentos legales, que se encargan de la protección del conocimiento fundamentados en "derechos de propiedad intelectual", a otra estrategia ofensiva basada en redes científicas (y de innovación) flexibles y dinámicas que tienen la evidente ventaja de desarrollar ciencia "justo a tiempo" y de poder llegar rápidamente al mercado, mediante su pronta conversión en nuevos bienes y servicios.

Tal enfoque caracterizado por su exclusivismo, es sustituido por una estrategia moderna de In vestigación+Desarrollo+Innovación $(|+D+|)$ que engloba tres tipos de actividades:

a) Investigación básica, consistente en trabajos originales, experimentales o teóricos, que se emprenden para obtener nuevos conocimientos sobre los hechos y fenómenos observables.

b) Desarrollo Tecnológico o Investigación aplicada, que busca encontrar nuevos usos de la investigación básica para la solución de problemas específicos.

c) Innovación sustentada en el desarrollo tecnológico que, con base en los conocimentos existentes, se dirige a la creación de nuevos materiales, productos, dispositivos, procesos, sistemas o servicios.
En razón de la universalidad de esos tres tipos de actividades, en el naciente siglo XXI las empresas o individuos que se dedican a la I+D+l, deben tener necesariamente una integración vertical, utilizando tanto redes como desarrolladores que, por sí solos, representan una minúscula parte de la I+D+l que se despliega a nivel global.

Para Tascott y Williams (2007), este nuevo sistema tiene una gran ventaja:

La sabiduría convencional dice que las compañías innovan, diferencian y compiten logrando buenos resultados. Se contratan y se mantienen a las "mejores personas" para generar nuevas ideas, hacer descubrimientos nuevos, competir y expandir sus líneas de negocios. Se escuchan a sus clientes y protegen su propiedad intelectual tenazmente. Se piensa globalmente pero se actúa de manera local, y se garantiza una buena ejecución gracias a su buena administración y sus eficientes controles (p. 22)

Una evidente ventaja adicional, es la de poder contactar, a través de la red Internet, a los científicos e innovadores de cualquier parte del planeta y tener acceso a la información de manera instantánea.

La Era de los Laboratorios de Investigación monolíticos y cerrados de las grandes empresas transnacionales, estaría así dejándole lugar a una completa apertura para todos aquellos profesionales que desean dedicarse tiempo completo o parcial a la I+D+I. Así, y siguiendo nuevamente a Tascott y Williams (2007):

En el siglo XXI está emergiendo una nueva clase de desarrollo empresarial, un tipo que abre sus puertas al mundo entero, acudiendo a múltiples fuentes de innovación, fundamentándose, especialmente en el criterio de sus clientes, compartiendo recursos que anteriormente estaban vedados, y acudiendo a los medios de comunicación masiva 
(especialmente Internet) en busca de nuevas ideas, rompiendo así con el comportamiento tradicional de las multinacionales $y$, en consecuencia, convirtiéndose en empresas globalizadas. Este nuevo sistema de operación se basa en cuatro nuevas ideas: apertura, compañerismo, colaboración y globalización" (p.37).

Esta nueva estrategia de I+D+I tiene las siguientes particularidades:

1. Cambia de forma radical no solo la creación sino también la fabricación, mercadeo y distribución de los bienes y servicios.

2. Abre la competitividad a niveles cada vez más globales, en beneficio de los investigadores y desarrolladores particulares.

3. El costo sufre una baja sustancial en beneficio directo de los consumidores;

4. Requiere de una nueva reglamentación internacional sobre propiedad intelectual y derechos de autor.

5. Podría representar una alternativa de disminuir la "ventaja comparativa" que tienen las transnacionales en beneficio directo de los países en vías de desarrollo.

\section{El contraste entre países desarrolados y en vías de desarrollo}

Existe un enorme contraste entre lo que invierten en I+D, los países desarrollados en relación con lo correspondiente de los países en vías de desarrollo.

Según datos de la UNESCO, (2010); para el año 2007, las cuatro potencias económicas del mundo invertían en I+D un promedio de un 3\% del PIB pero de forma un tanto desigual:
1. China con un 4,3

2. Japón con un 3,4

3. Estados Unidos un 2,6

4. Unión Europea con un 2,2

Otros de los países que más invirtieron en Investigación y Desarrollo, en proporción a su PIB, se muestra en la tabla 1:

TABLA 1

Gasto en I+D

\begin{tabular}{|l|c|}
\hline \multicolumn{1}{|c|}{ País } & \% del PIB para I+D \\
\hline Israel & 4,5 \\
\hline Suecia & 3,6 \\
\hline Finlandia & 3,5 \\
\hline Corea del Sur & 3,2 \\
\hline Islandia & 2,8 \\
\hline Austria & 2,6 \\
\hline Dinamarca & 2,6 \\
\hline Alemania & 2,5 \\
\hline
\end{tabular}

Fuente: elaboración propia con base en datos de UNESCO (2010).

No obstante se debe aclarar que en cuanto al total de gastos en I+D, los Estados Unidos siguen a la cabeza con US\$343 billones, seguido muy de largo por la Unión Europea con US\$231 billones, China con US\$136 billones, y Japón con US\$130 billones.

En el siguiente gráfico se puede apreciar, para estos países, la evolución del gasto desde 1998 al año 2007, último del que se tienen datos oficiales: 
FIGURA 1

\section{Porcentaje del Gasto en I+D en relación con el PIB}

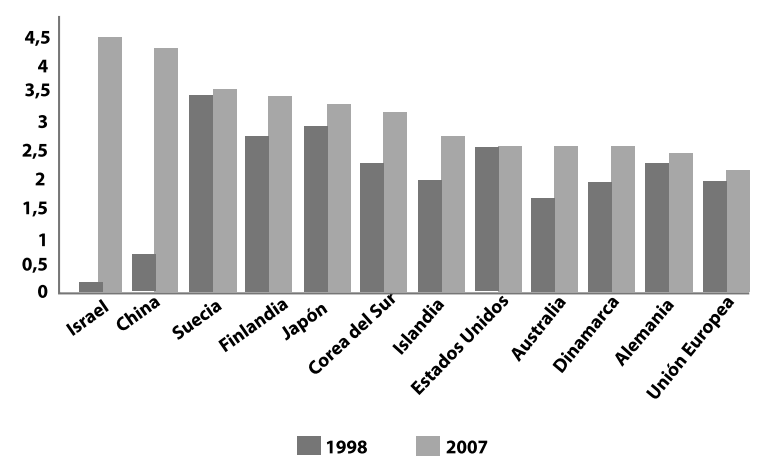

Fuente: elaboración propia con base en datos recopilados en UNESCO, 2010.

Es notorio el esfuerzo que han hecho países como Corea del Sur, Islandia y China; en tanto países desarrollados, en particular Estados Unidos y la Unión Europeas apenas mantienen sus niveles de 1998.

En cuanto a las áreas de investigación, a nivel mundial se invertía fundamentalmente en:

\section{TABLA 2}

\section{Inversión en I+D por rubros}

\begin{tabular}{|l|c|}
\hline \multicolumn{1}{|c|}{ Rubro } & \% del total en I+D \\
\hline Farmacia y biotecnología & 19,3 \\
\hline Informática: hardware & 17,6 \\
\hline Automóviles & 16,6 \\
\hline Electrónica & 7,4 \\
\hline Informática: software & 7,3 \\
\hline Química & 4,7 \\
\hline Defensa y aeroespacial & 4,4 \\
\hline Productos de lujo & 3,9 \\
\hline Ingeniería Industrial & 2,5 \\
\hline Industria general & 2,4 \\
\hline
\end{tabular}

Fuente: elaboración propia con base en datos recopilados en UNESCO (2010).
Nótese como cerca del 54\% se concentra en áreas de amplio desarrollo tecnológico: Farmacia y biotecnología, Informática y Automóviles.

En la tabla 3 se observa el caso de Costa Rica, un país en vías de desarrollo:

TABLA 3

\section{Inverción de Costa Rica por rubros}

\begin{tabular}{|l|c|}
\hline \multicolumn{1}{|c|}{ Rubro } & \% del total de I+D \\
\hline $\begin{array}{l}\text { Ciencias Naturales y } \\
\text { Exactas }\end{array}$ & 27,8 \\
\hline Ciencias Agrícolas & 26,7 \\
\hline Ciencias Sociales & 26,0 \\
\hline Ingeniería y Tecnología & 16,1 \\
\hline Ciencias Médicas & 2,5 \\
\hline Humanidades & 0,9 \\
\hline Total & 100,0 \\
\hline Alemania & 2,5 \\
\hline
\end{tabular}

Fuente: MCYT, publicado en el 2009.

Contrasta evidentemente con los datos anteriores pues también casi el $54 \%$ del total invertido en Costa Rica es destinado a Agricultura y Ciencias Sociales, áreas con bajo desarrollo tecnológico.

Otro significativo contraste, resulta en cuanto al Sector Inversor que desarrolla la I+D, es notable que en los países desarrollados la relación es 2:1 entre las empresas privadas y el Gobierno. Así en la UE esa relación porcentual es 55:33; en tanto que en los Estados Unidos es 66:27.

En contraste, el gasto en I+D de Costa Rica, corresponde casi en un 90\% al esfuerzo estatal, correspondiendo al Gobierno un 26\% y a las universidades estatales con un 62\%. 


\section{¿Qué sucede con los países en vías de desarrollo de América Latina?}

Corea del Sur invierte más del 3\% de su PIB en I+D seguido de cerca por Islandia. Ambos países con niveles muy semejantes al promedio de inversión de los países desarrollados.

En contraste, véase el caso de los países latinoamericanos y cómo distan tanto de los países citados que invierten más en I+D:

TABLA 4

\section{Inversión países de América Latina}

\begin{tabular}{|l|c|}
\hline \multicolumn{1}{|c|}{ País } & \% del PIB para I+D \\
\hline Brasil & 1,0 \\
\hline México & 0,5 \\
\hline Argentina & 0,5 \\
\hline Chile & 0,3 \\
\hline Costa Rica & 0,3 \\
\hline
\end{tabular}

Fuente: UNESCO, Instituto de Estadísticas, 2010.

Un dato más relevante es el gasto por habitante y la situación es la siguiente (Tabla 5).
TABLA 5

Gasto per cápita

\begin{tabular}{|l|c|}
\hline \multicolumn{1}{|c|}{ País } & Gasto/cápita \\
\hline Brasil & 91,6 \\
\hline Argentina & 67,3 \\
\hline México & 56,8 \\
\hline Chile & 34,6 \\
\hline Costa Rica & 28,7 \\
\hline
\end{tabular}

Fuente: UNESCO, Instituto de Estadísticas, 2010.

Es así como la diferencia entre los propios países latinoamericanos se hace abismal al comparar los datos del gasto per cápita pues Brasil supera en más de tres veces al país de la tabla que menos invierte: Costa Rica.

No obstante, no solo es bueno compararse consigo mismo sino también con los países más exitosos, en este caso de América, por lo que resulta conveniente presentar los datos de los tres "gigantes" de América Latina contrastándolos con los de Estados Unidos; a saber:

TABLA 6

\section{Comparativo países de América Latina con Estados Unidos}

\begin{tabular}{|c|c|c|c|c|c|c|}
\hline \multirow{2}{*}{ País } & \multicolumn{2}{|c|}{$\begin{array}{c}\text { Gasto en billones } \\
\text { de dólares }\end{array}$} & \multicolumn{2}{c|}{$\%$ del PIB } & \multicolumn{2}{c|}{ Gasto per cápita } \\
\cline { 2 - 7 } & $\mathbf{2 0 0 2}$ & $\mathbf{2 0 0 7}$ & $\mathbf{2 0 0 2}$ & $\mathbf{2 0 0 7}$ & $\mathbf{2 0 0 2}$ \\
\hline Argentina & 1,2 & 2,7 & $0,4 \%$ & $0,5 \%$ & 30,9 & 67,3 \\
\hline Brasil & 12,1 & 17,3 & $0,9 \%$ & $1,0 \%$ & 97,2 & 51,0 \\
\hline México & 4,2 & 5,9 & $0,4 \%$ & $0,5 \%$ & 56,8 \\
\hline E.U.A. & 277,1 & 368,8 & $2,7 \%$ & $2,7 \%$ & 952,7 & $1.205,9$ \\
\hline
\end{tabular}

Fuente: UNESCO, Instituto de Estadísticas, 2010. 
Se evidencia mejor si se grafica el dato más significativo: el gasto per cápita.

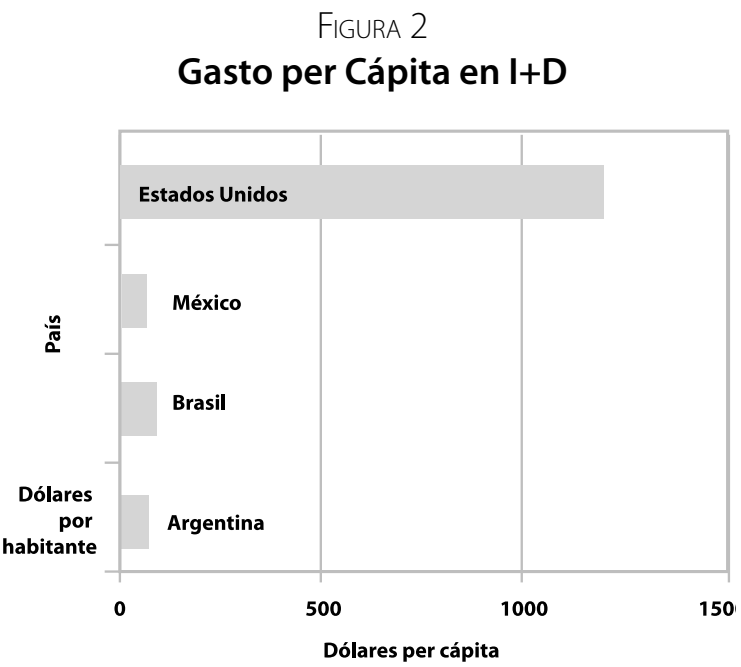

Fuente: elaboración propia con base en datos del Instituto de Estadística de la UNESCO (2010).

En promedio toda América Latina gastó en Actividades Científicas y Tecnológicas un 0,6\% del Producto Interno Bruto (PIB) en los años 2002 y 2007 respectivamente; en tanto que Estados Unidos lo hizo en un 2,7\% del PIB.

Por su parte, en el año 2007 Costa Rica gastó 327 millones de dólares en Actividades Científicas y Tecnológicas, es decir un 1,24\% del PIB pero únicamente 84 millones son destinados a I+D, lo que representa apenas un 0.32 del PIB, la mitad del porcentaje regional y la novena parte del de los Estados Unidos.

Ahora bien, ¿se refleja ese esfuerzo en los índices de crecimiento económico?; véase en la tabla 7 el resultado de esos mismos países en el período en estudio.

Nótese que más bien el comportamiento, es errático pues Costa Rica, el país que menos gasta es el que obtiene el segundo mayor crecimiento económico, en tanto que el que más gasta, Brasil, obtiene apenas un crecimiento medio (Tabla 8).
TABLA 7

Comparativo inversión en I+D/Crecimiento

\begin{tabular}{|l|c|c|}
\hline \multicolumn{1}{|c|}{ País } & Gasto/cápita & $\begin{array}{c}\text { Crecimiento } \\
\text { \% PIB }\end{array}$ \\
\hline Brasil & 91,6 & 4,0 \\
\hline Argentina & 67,3 & 7,6 \\
\hline México & 56,8 & 2,0 \\
\hline Chile & 34,6 & 4,0 \\
\hline Costa Rica & 28,7 & 5,5 \\
\hline
\end{tabular}

Fuente: elaboración propia con base en datos de Cepal, 2008.

Así, tratando de corroborar con respecto al gasto total:

TABLA 8

\section{Gasto en millones de dólares por país}

\begin{tabular}{|l|c|}
\hline \multicolumn{1}{|c|}{ País } & $\begin{array}{c}\text { Gasto total I+D } \\
\text { (millones de \$) }\end{array}$ \\
\hline Brasil & 13564 \\
\hline México & 3820 \\
\hline Argentina & 900 \\
\hline Chile & 764 \\
\hline C.osta Rica & 82 \\
\hline
\end{tabular}

Fuente: elaboración propia con base en datos de Cepal, 2008.

Nuevamente países que gastan relativamente poco, Argentina y Costa Rica, obtienen mejores resultados que el que más invierte, Brasil.

Obviamente esta relación $1+D$ vs. crecimiento económico, amerita un análisis ulterior cuya profundidad rebasa las posibilidades de este artículo.

\section{El conocimiento compartido}

Por años, las empresas compraban el conocimiento o lo adquirían a través de alianzas estratégicas, joint ventures o subcontrataciones; todo 
con el ánimo de cumplir con el objetivo primordial de toda empresa: incrementar la riqueza de sus accionistas.

En la actualidad, en razón -entre otros factores- del dinamismo introducido en el entorno económico por la red Internet, estos instrumentos convencionales resultan demasiado rígidos y no lo suficientemente accesibles como para permitirle a la empresa, por poderosa que fuera, crecer lo suficiente en I+D+l para mantener su competitividad; sobre todo ante la realidad de la globalización.

Con la intención de poder superar esta desventaja, las empresas acuden a las redes abiertas de Internet, donde pueden a menor costo, adquirir las innovaciones y desarrollos de investigadores y científicos de prácticamente cualquier parte del mundo. Tales redes son conocidas como Ideágoras. Su nombre proviene del "ágora" griego; una especie de plazas adonde los griegos intercambiaba no solamente bienes sino también ideas.

Estas redes constituyen en realidad una lluvia universal de ideas conformando un mercado de ideas excepcionales; las que son evaluadas por las diferentes empresas para determinar cuáles son viables de desarrollar y, en consecuencia, adquirir los derechos de propiedad intelectual correspondientes.

Tales ideas son desarrolladas para hacerlas llegar, en forma de bienes y servicios, a los consumidores que incrementarán su nivel de satisfacción.

Se estaría desarrollando así una nueva división del trabajo técnico-científico en donde la investigación y la innovación correrían por cuenta de individuos y microempresas y el desarrollo a cargo de las grandes empresas en razón de su capacidad financiera, de convertir las ideas en la creación o modificación de bienes y servicios para su posterior producción y distribución.

Para los países en vías de desarrollo en general y para los de América Latina en particular, po- dría suponer una ventaja comparativa el poder aprovechar los escasos recursos que se destinan a la investigación e innovación, organizando redes inteligentes (ideágoras) que trasciendan las fronteras unificando esfuerzo latinoamericano, a cargo fundamentalmente de las universidades públicas, para crear un banco de ideas innovadoras y ofrecérselas a la empresa privada (nacional o extranjera) para su posterior desarrollo en forma de nuevos (o modificados) bienes y servicios.

Conviene recordar que en América Latina, gracias fundamentalmente al apoyo oficial, se han desarrollado varias redes tecnológicas y científicas (Anexo 1), las cuales servirían de muy buena base para iniciar el proceso de integración en pos de una mejor utilización de los recursos destinados a la investigación e innovación.

En la tabla 9, se muestra que el esfuerzo primordial es del Sector Estatal, muy al contrario al caso de los Estados Unidos donde la mayor inversión lo hace la empresa privada:

TABLA 9

\section{Gasto en I+D según Sector}

\begin{tabular}{|l|c|c|}
\hline Gasto por sector & América Latina & $\begin{array}{c}\text { Estados } \\
\text { Unidos }\end{array}$ \\
\hline Gobierno & $56,90 \%$ & $30 \%$ \\
\hline Empresas & $37,20 \%$ & $64,60 \%$ \\
\hline $\begin{array}{l}\text { Educación } \\
\text { Superior }\end{array}$ & $4,4, \%$ & $2,7 \%$ \\
\hline $\begin{array}{l}\text { Organizaciones } \\
\text { privadas sin fines } \\
\text { de lucro }\end{array}$ & $0,40 \%$ & $2,70 \%$ \\
\hline Extranjero & $1,10 \%$ & --- \\
\hline
\end{tabular}

Fuente: Unesco, Instituto de Estadísticas, 2010.

Resulta fácil colegir de los datos anteriores, que en América Latina la I+D+I, queda en manos de las universidades públicas y, en cuanto financiadas por el Estado, en última instancia de los gobiernos de turno; los cuales casi nunca se rigen 
por criterios objetivos en la asignación de los recursos financieros por lo que, en definitiva, el importante rubro de la investigación, el desarrollo y la innovación queda en manos de voluntades más políticas que técnicas, con las subsecuentes consecuencias en cuanto a la cantidad y la calidad del quehacer científico.

De ahí, la importancia de que las incipientes redes latinoamericanas de ciencia y tecnología, se constituyan como organizaciones independientes sin fines de lucro y con facultades suficientes para poder concretar alianzas estratégicas y hasta fusiones con sus adláteres.

\section{CONCLUSIÓN}

Las redes científicas constituyen una buena opción para los países en vías de desarrollo para accesar a los esfuerzos que hace la comunidad internacional, sobre todo, en I+D.

El problema estriba en que sigue existiendo una diferencia abismal entre la inversión de estos país en relación con lo que gastan los países desarrollados.

Lo anterior se agrava con el hecho de que también hay una diferenciación cualitativa del gasto, pues los países desarrollados invierten en actividades que al fin y al cabo le van a significar mayor rentabilidad por su valor agregado. Sin duda que la razón de esta particular estrategia, estriba en que son las empresas las que más invierten en I+D, en tanto que en el subdesarrollo lo hacen los estados; por lo general, a través de los centros universitarios estatales.

Otra diferencia importante está en la propia estructura del concepto pues las posibilidades de los países ricos están más en el desarrollo de las innovaciones científicas y, en consecuencia, en su capacidad de convertirlas en ventajas comerciales.

Es importante que los países en vías de desarroIlo, redireccionen su esfuerzo hacia el concepto de I+I en vez de I+D.
Se le da así mayor relevancia a la innovación en vez del desarrollo, pues este último requiere inversiones mucho mayores que las de la investigación e innovación de bienes y servicios.

Al respecto hay que tomar en consideración que la innovación tecnológica es en la actualidad, la más importante fuente de cambio en la cuota de mercado entre firmas competidoras y el factor más frecuente en la desaparición de las posiciones consolidadas.

Existe, por otra parte, en el caso de los países latinoamericanos, el problema de la dependencia financiera con respecto al gobierno central y la consecuente influencia que pueda ejercer la política en decisiones que, se supone, deben caracterizarse por una completa objetividad científica. De ahí la propuesta de reorientar su organización hacia entidades independientes y con completa autonomía en la toma de decisiones.

\section{REFERENCIAS}

CEPAL (2008). Estudio Económico de América Latina 2007-2008. Recuperado el 3-03-11 de: http://www. eclac.org/cgi-bin/getProd.asp?xml=/publicaciones/ xml/4/36464/P36464.xml\&xsl=/de/tpl/p9f.xsl\&base=/ tpl/top-bottom.xslt.

Ministerio de Ciencia y Tecnología (2008). Indicadores nacionales de ciencia, tecnología e innovación: indicadores nacionales 2006-2007. San José: MICIT. Recuperado el 03-03-2011 de: http://www.micit.go.cr/ index.php/docman/doc_details/77-indicadores-nacionales-2006-2007-ciencia-tecnologia-e-innovacioncosta-rica.html

Red de Indicadores de Ciencia y Tecnología Iberoamericana e Interamericana (2007). Estadísticas Centrales de Ciencia y Tecnología. San José: MICIT

Tascott, D. \& William, A. (2007). Wikinomics. New York: Atlantic Books.

UNESCO (2010). Informe de la UNESCO sobre la Ciencia 2010. París, Francia. Ediciones UNESCO.

Recibido: 03 de junio de 2010 Aceptado: 15 de enero de 2011 
RNA Revista Nacional de Administración

\section{Redes Tecnológicas y Científicas de América Latina}

\begin{tabular}{|c|c|c|}
\hline País & Red & Ente rector \\
\hline ARGENTINA & Red Nacional de Investigación y Educación RETINA & $\begin{array}{l}\text { Secretaría de Ciencia y Tecnología } \\
\text { (SECYT) }\end{array}$ \\
\hline BOLIVIA & Red Boliviana de Comunicación de Datos BOLNET & $\begin{array}{l}\text { Consejo Nacional de Ciencia y } \\
\text { Tecnología (CNCT) }\end{array}$ \\
\hline BRASIL & Rede Nacional de Ensino e Pesquisa RNP & Consejo de Ciencia y Tecnología (CCT) \\
\hline CHILE & Red Universitaria Nacional REUNA & $\begin{array}{l}\text { Consejo Nacional de Ciencia y } \\
\text { Tecnología (CONICYT). }\end{array}$ \\
\hline \multirow{2}{*}{ COLOMBIA } & Red Hemisférica Universitaria de Ciencia y Tecnología & \multirow{2}{*}{$\begin{array}{l}\text { Consejo Nacional de Ciencia y } \\
\text { Tecnología (CONACYT) }\end{array}$} \\
\hline & HUCYT & \\
\hline COSTA RICA & Red Nacional de Investigación CRNET & $\begin{array}{l}\text { Ministerio de Ciencia y Tecnología } \\
\text { (MICIT). }\end{array}$ \\
\hline \multirow{2}{*}{ CUBA } & Red Cubana de la Ciencia & \multirow{2}{*}{$\begin{array}{l}\text { Ministerio de Ciencia, Tecnología y Medio } \\
\text { Ambiente (CITMA). }\end{array}$} \\
\hline & RCC & \\
\hline \multirow{2}{*}{ ECUADOR } & Red Ecuatoriana de Ciencia y Tecnología & \multirow{2}{*}{$\begin{array}{l}\text { Secretaria Nacional de Ciencia y } \\
\text { Tecnología (SENACYT). }\end{array}$} \\
\hline & REICYT & \\
\hline \multirow{2}{*}{ EL SALVADOR } & Red Nacional de Investigación y Educación & \multirow{2}{*}{$\begin{array}{l}\text { Consejo Nacional de Ciencia y } \\
\text { Tecnología CONACYT }\end{array}$} \\
\hline & RAICES & \\
\hline \multirow{2}{*}{ HONDURAS } & Red Hemisférica Universitaria de Ciencia y Tecnología & \multirow{2}{*}{$\begin{array}{l}\text { Consejo Hondureño de Ciencia y } \\
\text { Tecnología (COHCIT). }\end{array}$} \\
\hline & HUCYT & \\
\hline MEXICO & $\begin{array}{l}\text { Corporación Universitaria para el Desarrollo de Internet } \\
\text { CUDI }\end{array}$ & $\begin{array}{l}\text { Consejo Nacional de Ciencia y } \\
\text { Tecnología (CONACYT). (SES }\end{array}$ \\
\hline NICARAGUA & Red de Ciencia y Desarrollo & $\begin{array}{l}\text { Consejo Nicaragüense de Ciencia } \\
\text { y Tecnología (CONICYT). }\end{array}$ \\
\hline PANAMA & Red de Ciencia y Tecnología REDCYT & $\begin{array}{l}\text { Secretaría Nacional de Ciencia y } \\
\text { Tecnología (SENACYT). }\end{array}$ \\
\hline PARAGUAY & Red Nacional de Innovación & $\begin{array}{l}\text { Consejo Nacional de Ciencia y } \\
\text { Tecnología (CONICYT). }\end{array}$ \\
\hline \multirow{2}{*}{ PERU } & Red Internacional de Ciencia y Tecnología & \multirow{2}{*}{$\begin{array}{l}\text { Consejo Nacional de Ciencia y } \\
\text { Tecnología (CONCYTEC). }\end{array}$} \\
\hline & RICT & \\
\hline $\begin{array}{l}\text { REPÚBLICA } \\
\text { DOMINICANA }\end{array}$ & (SEESCYT). (CONESCT) & (SEESCYT). (CONESCT) \\
\hline \multirow{2}{*}{ URUGUAY } & Red Especial Uruguaya & \multirow{2}{*}{ (MEC). (DINACYT) (CONICYT). } \\
\hline & REU & \\
\hline \multirow{2}{*}{ VENEZUELA } & Red Académica Universitaria & \multirow{2}{*}{$\begin{array}{l}\text { Ministerio de Ciencia y Tecnología } \\
\text { (MICYT) }\end{array}$} \\
\hline & REACCIUN & \\
\hline
\end{tabular}

Fuente: elaboración propia con base en datos de cada país.

1342 (1), Enero - Junio, 2011 